\title{
Physical Exam Result
}

National Cancer Institute

\section{Source}

National Cancer Institute. Physical Exam Result. NCI Thesaurus. Code C83119.

The outcome of the physical examination assessment. 